\title{
Advantages and prospects of optical coherence tomography in interventional therapy of coronary heart disease (Review)
}

\author{
JIE WANG $^{1 *}$, SHUO YUAN ${ }^{2,3^{*}}$, JINGJING QI $^{1}$, QINGGAO ZHANG ${ }^{3}$ and ZHENG JI ${ }^{1}$ \\ ${ }^{1}$ Department of Cardiology, Tangshan Gongren Hospital Affiliated of North China University of Science and Technology, \\ Tangshan, Hebei 063000; ${ }^{2}$ Key Laboratory of Natural Medicines of The Changbai Mountain, Ministry of Education, \\ College of Pharmacy, Yanbian University, Yanji, Jilin 133002; ${ }^{3}$ Chronic Diseases Research Center, Medical College, \\ Dalian University, Dalian, Liaoning 116622, P.R. China
}

Received August 19, 2021; Accepted January 13, 2022

DOI: $10.3892 / \mathrm{etm} .2022 .11180$

\begin{abstract}
Coronary heart disease is the leading cause of mortality among all diseases globally. Percutaneous coronary intervention (PCI) is a key method for the treatment of coronary heart disease. Optical coherence tomography (OCT) is an optical diagnostic technology with a resolution of up to $10 \mu \mathrm{m}$, which is able to accurately assess the composition of the coronary arterial wall and determine the characteristics of atherosclerotic lesions. It is also highly consistent with pathological examinations, optimizing the effect of stent implantation and evaluation of the long-term effectiveness and safety of the stent, which has irreplaceable value in the field of precision and optimization of coronary intervention. The innovative OCT technology may help provide more comprehensive clinical research evidence. The application of OCT in clinical and basic research of coronary atherosclerosis, selection of treatment strategies for acute coronary syndromes, optimization of interventional treatment efficacy, evaluation of novel stents, intimal stent coverage and selection of dual antiplatelet drugs has become more widely used, affecting the current coronary interventional treatment strategies to a certain extent. The aim of the present review was to discuss the role of OCT in evaluating preoperative plaque characteristics,
\end{abstract}

Correspondence to: Dr Zheng Ji, Department of Cardiology, Tangshan Gongren Hospital Affiliated of North China University of Science and Technology, 27 Wenhua Road, Lubei, Tangshan, Hebei 063000, P.R. China

E-mail: jizheng999@163.com

Dr Qinggao Zhang, Chronic Diseases Research Center, Medical College, Dalian University, 10 Xuefu Street, Economic and Technological Development Zone, Dalian, Liaoning 116622, P.R. China

E-mail: zhangqinggao@dlu.edu.cn

${ }^{*}$ Contributed equally

Key words: optical coherence tomography, coronary heart disease, vulnerable plaque, percutaneous coronary intervention guiding PCI and evaluating the effects of postoperative stents or drug treatments.

\section{Contents}

1. Introduction

2. OCT evaluation of atherosclerotic plaque-related factors

3. OCT guides interventional coronary artery treatment

4. OCT guides complex lesion management

5. Application of OCT in the post-stent implantation follow-up

6. Factors and common problems affecting OCT imaging results

7. Application prospects of OCT in the field of cardiovascular interventional diagnosis and treatment

8. Summary

\section{Introduction}

In heart disease, medical imaging may provide an objective basis for the diagnosis and suitable treatment of changes in cardiac structure and minor pathological changes. The imaging examination techniques of the coronary arterial lumen mainly include coronary angiography (CAG), intravascular ultrasound (IVUS), optical coherence tomography (OCT), angioscopy and near-infrared spectroscopy (NIRS), which are able to clearly display the internal structure and pathological changes of blood vessels (1). These provide clinicians with real-time dynamic observations, help reach the correct diagnosis of the disease in a timely manner and provide reasonable treatment strategies (Table I).

CAG is a routine method used to assess the extent and severity of coronary artery disease. Intracoronary imaging methods, such as IVUS and OCT, may provide helpful information on the morphology of the coronary arteries and may help evaluate the size of the lumen, plaque load and composition in detail, which are helpful for treatment planning (1). As presented in Table II, accumulating evidence suggests that both IVUS and OCT are able to optimize the deployment and outcome of the stent while improving patient prognosis, particularly regarding the prognostic significance of the 
disease (the left main disease) and anatomical structures that are difficult to assess on imaging $(2,3)$.

\section{OCT evaluation of atherosclerotic plaque-related factors}

Atherosclerotic plaque is a term used to describe a space-occupying lesion (thickening lesion) or loss of a three-layer structure in the blood vessel wall on OCT images. The OCT diagnostic criteria stated below refer to the internationally recognized OCT consensus (4-6).

Types of plaques on OCT images. In the OCT image, the normal coronary artery wall is characterized by a typical three-layer structure (Fig. 1A), which is composed of blood intima, media and adventitia (Fig. 1B). The intima of the blood vessel has a high reflection signal, the reflection signal of the media is usually low or weak and the adventitia frequently exhibits an uneven high-reflection signal. In OCT, the internal elastic membrane (IEM) is defined as the boundary between arterial intima and media, while the external elastic membrane (EEM) is defined as the boundary between arterial media and adventitia (Fig. 1B). Compared with traditional coronary angiography, OCT has marked high-resolution characteristics. OCT is able to accurately identify certain atherosclerotic plaques, adventitia, EEM and IEM, and may accurately identify different tissue characteristics of plaques, including fibers (Fig. 1C), calcification components (Fig. 1D) and lipids (Fig. 1E). At the same time, the evaluation of the microstructure in the plaque by OCT is unmatched by other imaging techniques, including cholesterol crystals (Fig. 1F), macrophages (Fig. 1G), microvessels (Fig. 1H) and microcalcifications. These types of plaques have differences in scattering characteristics (intensity of the reflected signal) and attenuation characteristics (as the signal penetrates deeper into the tissue layer, the intensity of the light signal gradually decreases through scattering and absorption). Therefore, these plaques with different properties will have corresponding characteristic changes on the OCT image. As is presented in Fig. 1 and Table III, the plaques displayed in OCT images include fibrous, calcified and lipid plaques; microstructures within the plaques may also be observed (5-7).

Vulnerable plaque. As early as 1989, Muller et al (8) put forward the concept of 'vulnerable plaque' to study acute cardiovascular disease. According to that study, the leading cause of acute coronary syndrome (ACS) is the rupture of an unstable plaque and secondary thrombosis. Kolodgie et al (9) renamed the 'vulnerable plaque' to 'thin-cap fibroatheroma' (TCFA) after combining the autopsy results of patients who died from cardiac causes. This term refers explicitly to an atherosclerotic plaque with macrophage infiltration and a necrotic center covered by a fibrous cap with a thickness of $\leq 65 \mu \mathrm{m}$ (Fig. 2). As presented in Table IV, Naghavi et al (10) provided the histopathological definition and diagnostic criteria of vulnerable plaque (10-12).

A previous study used OCT to perform imaging analysis on 26 cases with ST-segment elevation myocardial infarction (STEMI) and 16 patients with stable angina pectoris. The results indicated that patients with STEMI had a higher proportion of TCFAs observed in the 'culprit lesions' ( 85 vs.
$13 \%$, respectively; $\mathrm{P}<0.001$ ), while the fibrous cap was thinner (57 \pm 12 vs. $180 \pm 65 \mu \mathrm{m}$, respectively; $\mathrm{P}<0.001$ ) (13). In addition to TCFA, plaque rupture, plaque erosion, calcified nodules, macrophage infiltration and formation of nutrient blood vessels are all associated with the occurrence of ACS. Thus, the evaluation and quantitative analysis of vulnerable plaques may guide the risk stratification of clinical conditions, as well as the protection and treatment of vulnerable plaques, and help evaluate treatment efficacy.

Advantages and disadvantages of methods used for evaluating vulnerable plaques. In the clinic, the coronary angiography results of numerous patients are not able to display the underlying coronary artery lesions, particularly culprit lesions that are not able to identify thrombosis, whether they are caused by unstable plaque rupture, plaque erosion or calcified nodules. As presented in Table $\mathrm{V}$, although several methods may be used to detect vulnerable plaques, various detection methods have their advantages and disadvantages $(14,15)$. The major factor that determines the vulnerability of plaques is abnormal plaque structure and OCT is able to accurately identify plaque components and microstructure within the plaque due to its high resolution. The superficial calcification and neovascularization of the five main and secondary criteria for vulnerable plaques may be accurately analyzed qualitatively or quantitatively, making it the most ideal imaging technique for identifying vulnerable plaques.

\section{Identification of vulnerable plaques by $O C T$}

OCT measures the thickness of the fibrous cap and may identify the thin fibrous cap. The thickness of the fibrous cap is the decisive factor for vulnerable plaques (16). The fibrous cap appears as a high-density, low-reflection image on OCT. OCT may clearly distinguish vulnerable plaques with thin fibrous caps (Fig. 2A). The thickness of the fragile cap, which is widely accepted and recognized in clinical practice, is $65 \mu \mathrm{m}$ (17). TCFA, in the context of OCT, is defined as a lipid-rich plaque with a thin fibrous cap (the thinnest part is $\leq 65 \mu \mathrm{m}$ ), and the lipid component in a plaque occupies $\geq 2$ quadrants (17). A previous study performed a histomorphological analysis of 295 coronary plaques (105 fibrous plaques, 88 TCFAs and 102 ruptured plaques) in patients who suffered sudden cardiac death. That study indicated that fibrous cap thickness distinguishes these types of plaques. The critical feature of the TCFA is that the plaque volume increases and the stenosis of the coronary arterial lumen becomes increasingly severe, which may lead to plaque rupture, causing an acute coronary event (18).

Microchannels. A microchannel on OCT imaging is defined as a non-signal luminal structure image of $>3$ frames that does not communicate with the lumen (19). The greater the number of microchannels $(\geq 2)$, the higher the proportion of TCFAs and the plaques with microchannels are more likely to rupture (20). On the one hand, microchannels may provide nutrients and oxygen to the heart muscle; on the other hand, they may promote the influx of lipids and inflammatory cell infiltration in coronary plaques. The newly formed microchannels are fragile and easy to rupture, leading to hemorrhage in the plaque, which eventually leads to a rapid increase in plaque volume and narrowing of the lumen (20). 
Table I. Comparison of several catheter technologies.

\begin{tabular}{|c|c|c|c|c|c|}
\hline Item & CAG & OCT & IVUS & Angioscopy & NIRS \\
\hline Plaque volume & - & - & ++ & + & - \\
\hline Calcification & ++ & ++ & +++ & - & - \\
\hline Fiber cap & - & +++ & + & + & + \\
\hline Lipid core & - & +++ & ++ & + & ++ \\
\hline Inflammation & - & + & - & - & - \\
\hline Thrombus & + & ++ & + & +++ & + \\
\hline Vascular remodeling & - & - & ++ & - & - \\
\hline Stent expansion & - & ++ & ++ & + & - \\
\hline Intimal hyperplasia & + & ++ & + & + & - \\
\hline
\end{tabular}

OCT, optical coherence tomography; CAG, coronary angiography; IVUS, intravascular ultrasound; NIRS, near-infrared spectroscopy; -, no recognition ability; + , weak recognition; ++ , fair recognition ability; +++ , strong recognition.
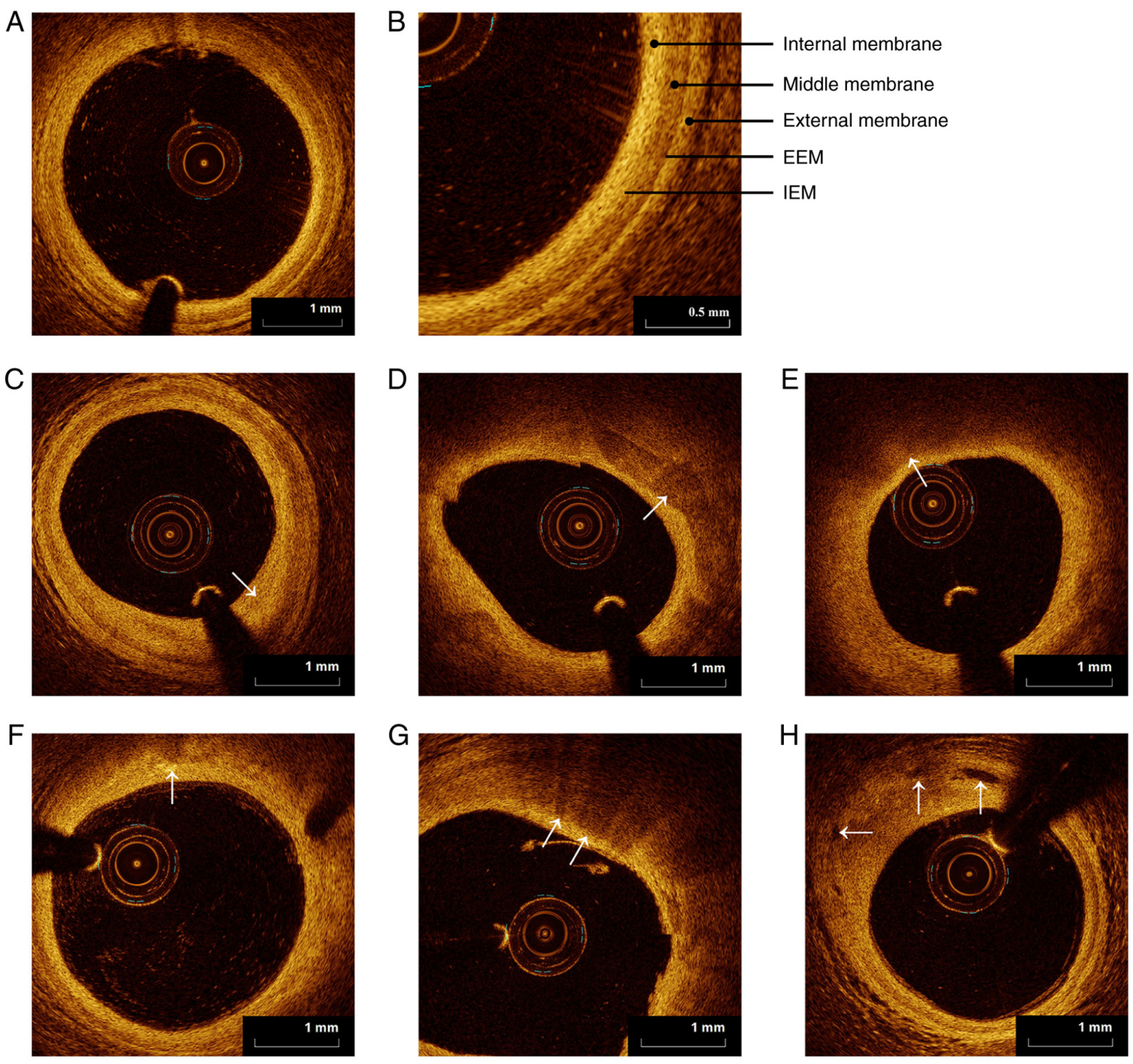

Figure 1. Types of plaques in optical coherence tomography images. (A) Diagram of normal coronary arteries (scale bar, $1 \mathrm{~mm}$ ). (B) Magnified image of normal coronary artery wall (scale bar, $0.5 \mathrm{~mm}$ ). Representative images of (C) fibrous plaque, (D) calcified plaque, (E) lipid plaque, (F) cholesterol crystal, (G) macrophage infiltration and (H) microchannel (arrows, scale bar, $1 \mathrm{~mm}$ ). EEM, external elastic membrane; IEM, internal elastic membrane.

Rupture and erosion of the plaques. On OCT, plaque rupture (Fig. 2B) manifests as intimal tearing, destruction or plaque fibrous cap dissection. Erosion (Fig. 2C) refers to an irregular or discontinuous vascular intimal surface with 
Table II. Comparison of the characteristics of OCT and IVUS.

A, IVUS modality

Advantages

Limitations

The development of IVUS and the recognition of images are relatively mature

IVUS is able to clearly display the intravascular structure and evaluate the plaque composition It may optimize and guide the treatment of coronary intervention

It may be used to study the mechanism of plaque progression or regression prior to and after treatment, vascular remodeling and restenosis after stent implantation

\section{B, OCT modality}

It has an ultra-high resolution comparable to histology, allowing real-time observation of small structures and lesions in blood vessels

It is able to accurately measure coronary luminal parameters, observe the morphological characteristics of arterial intima and plaques, and identify vulnerable plaques and macrophage infiltration prior to surgery

It is able to make the percutaneous coronary intervention process more refined and optimize the immediate effect after stent implantation. It may accurately observe the postoperative situation of various types of stent implantation at the cross-sectional level
The currently developed IVUS imaging catheter has poor passing ability for more severe stenosis or twisted angular lesions

As different tissues may have the same acoustic properties, the same density value may be reflected in IVUS images The current IVUS on the market has a low resolution and is not able to make accurate judgments for small structures and lesions in blood vessels, and corresponding changes in the lumen after stent implantation

cross-sectional level

(n)
The OCT imaging process requires blocking or removal of the blood in the corresponding detection vessel, which increases the difficulty of the operation and limits its application in severe coronary ischemic diseases OCT has a weak penetrating ability and is not able to accurately analyze the whole picture of the lesion. It is also difficult to measure the diameter of larger blood vessels and evaluate vascular remodeling
OCT is not able to clearly identify the internal tissue covered by the thrombus (particularly red thrombus) inside the blood vessel

In the detection after stent implantation, although OCT may clearly detect the stent intima, it is still unable to distinguish the histological characteristics of the stent surface covering the intima

OCT, optical coherence tomography; IVUS, intravascular ultrasound.

a tiny thrombus; however, there is no fibrous cap damage or void formation (21). An OCT study systematically classified and defined ACS culprit plaques and indicated that plaque rupture, plaque erosion and calcified nodules under the OCT definition accounted for 43.7, 31.0 and $7.9 \%$ of culprit lesions in ACS (22).

Macrophages. Macrophage activity is a crucial feature for evaluating plaque stability. Macrophages appear as dot-shaped areas with abundant, apparent or fused signals on OCT and the noise intensity of this area is stronger than the noise intensity of background spots (21). It has been confirmed that macrophages are associated with the severity of clinical symptoms. A study used OCT to image culprit and non-culprit lesions in a group of patients with stable angina pectoris, unstable angina pectoris
(UAP) and STEMI and quantitatively analyzed macrophages in the plaques. The results suggested that the cell density in patients with STEMI and UAP was increased, the density of macrophages in the culprit plaque lesion was higher compared with that of the non-culprit lesion and the macrophage density in the ruptured plaque was greater compared with that of the non-ruptured plaque (23).

Calcified nodules. Calcified nodules appear as low-density areas with distinct edges, distinguished from the fuzzy edges of the fat nucleus (21). Among them, punctate calcification (calcification angle $<90^{\circ}$, length $<10 \mathrm{~mm}$ ) is considered to be one of the characteristics that affect plaque stability. A study using OCT to observe the association between punctate calcification and plaque vulnerability suggested that 


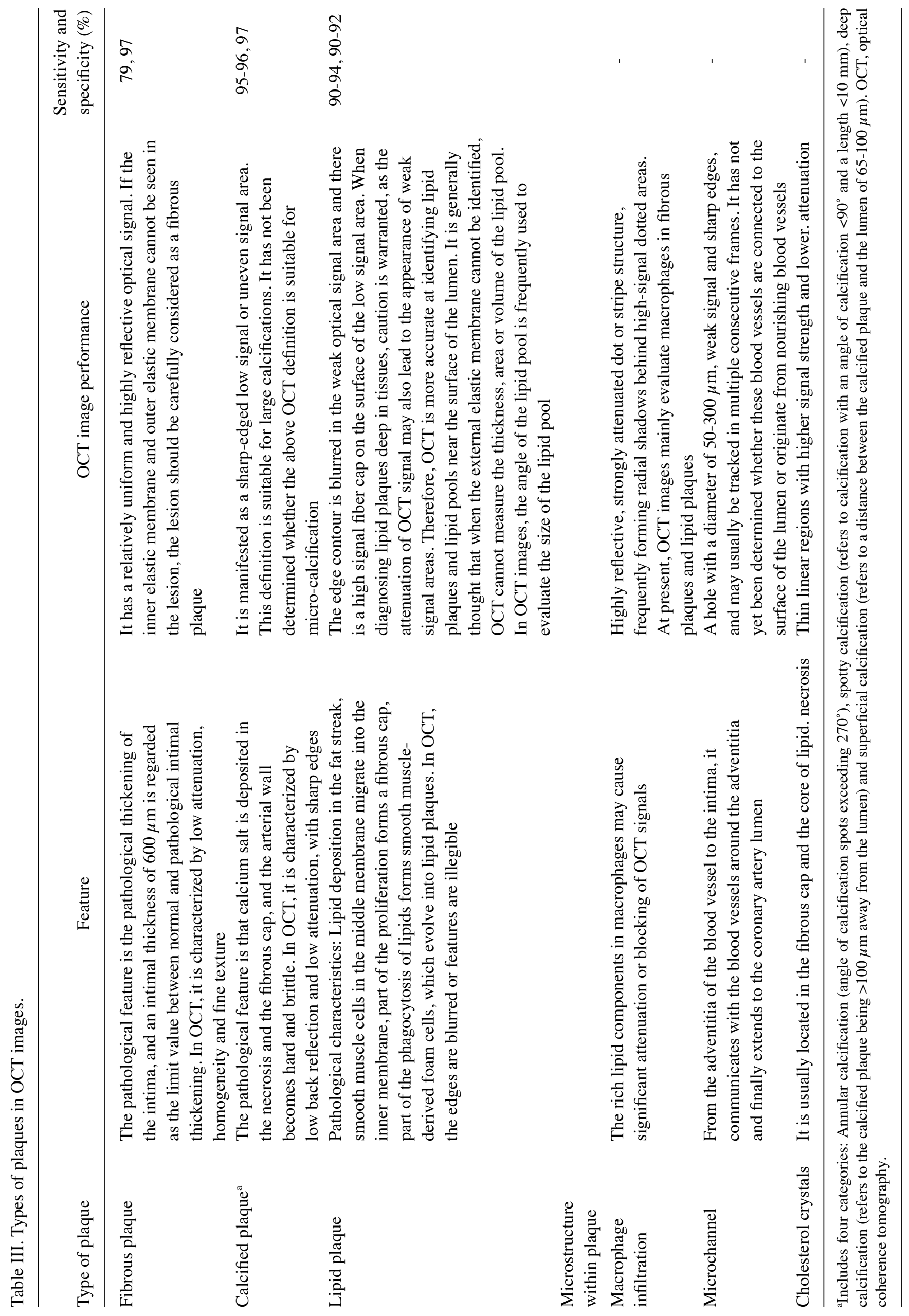


Table IV. Histopathological definition and criteria of vulnerable plaque (10-12). A positive diagnosis was made when one of the main criteria and at least two of the secondary criteria were met.

A, Main criteria

Histopathological feature

Definition and characterisation

Active inflammation

Thin fiber cap and large lipid core

Exfoliation of endothelium with

platelet aggregation

Damaged or cracked plaque

Severe stenosis $(>90 \%)$

Plaques with active inflammation frequently have a large number of monocytes, macrophages and $\mathrm{T}$ lymphocytes infiltrated and aggregated

The thickness of the fibrous cap of these plaques is $<100 \mu \mathrm{m}$ and the lipid core accounts for more than $40 \%$ of the total plaque volume

These plaques are characterized by superficial erosion with platelet aggregation or cellulose deposition

Most of them are recent ruptures, which may be the cause of subacute thrombosis

The surface shear force at severely narrowed areas has a vital role in the formation of thrombus and occlusion

B, Secondary criteria

Histopathological feature

Definition and characterisation

Superficial calcified nodules

There are calcified nodules in or near the fiber cap of the plaque. These nodules will highlight the plaque and cause the plaque to rupture. This may not be related to the severity of the calcification

Yellow shiny patches

The yellow plaques under angioscopy suggest large lipid cores and thin fibrous caps, which are easier to rupture, but this indicator lacks sufficient specificity

Bleeding in the plaque

Red blood cell overflow or iron deposits in the plaque may reflect the instability of the plaque

Endothelial dysfunction

Occurring in a variety of acute and chronic disease states, active inflammation and oxidative stress in vulnerable plaques are thought to be related to endothelial dysfunction

Positive remodeling of blood vessels

Studies have indicated that positive vascular remodeling is an important sign of vulnerable plaque

plaques containing punctate calcifications have more vulnerable plaque characteristics in patients with coronary heart disease. The number of punctate calcifications in the plaque is significantly correlated with the thickness of the fibrous cap; furthermore, the number of punctate calcifications in the plaque is also positively correlated with the incidence of microchannels (24).

Positive vascular remodeling. A study by Vink et al (25) confirmed that positive remodeling of the vascular lumen is related to the instability of the plaque; however, due to the low penetration of OCT, relevant studies are sparse.

Recognition of thrombus. OCT is able to recognize all types of thrombus and it is more sensitive and specific compared with IVUS (Fig. 2). OCT may also clearly distinguish between red and white thrombi in the lesion (21). The red thrombus is a strong backscatter and appears as a radially shaded area with a high signal on the surface and low or no signal posteriorly (Fig. 2D). The white thrombus is a low backscatter, showing a homogeneous region of high or normal signal with an irregular shape (Fig. 2E).

\section{OCT guides interventional coronary artery treatment}

OCT has significant advantages in evaluating acute lesions, guiding PCI and evaluating the mechanisms underlying treatment failure.

Assessment of borderline lesions. Regarding the diagnostic threshold of OCT for evaluating the degree of coronary artery stenosis and the treatment strategy under the corresponding threshold, there is still no consensus. A previous study compared the diagnostic efficacy of OCT and IVUS for coronary artery disease with fractional flow reserve $($ FFR) $\leq 0.8$ (26). The results suggested that, with a minimal luminal area (MLA) of $1.95 \mathrm{~mm}^{2}$ as the cutoff value, OCT has moderate diagnostic power, with a sensitivity of $82 \%$ and a specificity of $63 \%$. With an MLA of $2.63 \mathrm{~mm}^{2}$ as the cutoff value, the sensitivity decreases to $67 \%$ and the specificity is $65 \%$, and there is no statistically significant difference between OCT and IVUS in terms of diagnostic performance [area under the curve (AUC): 0.70, 95\% confidence interval (CI): $0.55-0.83$ to AUC: 0.63 , 95\% CI: 0.47-0.77; $\mathrm{P}=0.19]$. In the subgroup of small blood vessels (reference diameter 
Table V. Advantages and disadvantages of several methods for evaluating vulnerable plaques (14-15).

\begin{tabular}{lll}
\hline Modality $\quad$ Advantages & Disadvantages
\end{tabular}

CAG The method of CAG is simple, it requires a short time, The effect of foreshortening leads to underestimation has fewer complications and high diagnostic value. To a certain extent, the smoothness and stenosis of the lumen may be observed

Angioscopy The surface of the thrombus and plaque may be directly observed and the fibrous cap rupture and thrombosis may be detected. At the same time, the color of the plaque may be observed (white indicates mostly a stable plaque and yellow mostly a vulnerable plaque)

IVUS It is able to clearly distinguish the structure of each layer of the blood vessel wall, determine the diameter of the lumen, plaque volume, load and vascular remodeling, and may distinguish the properties of plaque such as lipid core, calcification and fibrous tissue

With strong penetrating power, it may provide overall and comprehensive imaging information for plaque assessment

OCT It has a high resolution, may accurately evaluate the microstructure close to the lumen, particularly in the unstable components of plaque, such as the thickness of the fiber cap, macrophage infiltration, lipid plaque size or plaque rupture. The fibrous cap thickness measurement is in good agreement with histology of the length of the stenosis of the lesion and there are large errors in the measurement of the vascular structure at the bifurcation and the eccentric plaque, and it is impossible to clearly determine whether the plaque is a vulnerable plaque

The size of the device is large. It may only be used to observe limited blood vessels and is not able to observe the inside of the plaque and the blood vessel wall. In addition, the blood flow requires to be blocked, which may cause remote ischemia

IVUS may only display image information of plaque subcomponents and not able to provide any quantitative detection. Only the calcified surface structure may be observed and the display effect is not optimal

The thickness of the fiber cap cannot be accurately determined and the detection of thrombus is not sufficiently sensitive

The penetration ability is weak (1-2 mm) and its penetration depth is far less than that of IVUS $(8-10 \mathrm{~mm})$. OCT is not able to provide an accurate analysis of the full picture of the lesion. The lesion area covered by the thrombus may not be used for an accurate assessment

OCT, optical coherence tomography; CAG, coronary angiography; IVUS, intravascular ultrasound.

$<3 \mathrm{~mm}$ ), OCT provided significant advantages (AUC: 0.77, 95\% CI: $0.60-0.89$ vs. AUC: 0.63 , 95\% CI: 0.46-0.78; $\mathrm{P}=0.04$ ). However, although OCT has higher diagnostic power compared with IVUS in this subgroup, its specificity for evaluating functional stenosis is low.

Furthermore, it is not sufficient to formulate a treatment strategy solely based on the degree of stenosis or the length of the lesion (27). The nature of the plaque frequently provides important information and it is also of far-reaching significance for guiding clinical treatment. The advantage of OCT lies in the accurate identification of thrombi, vulnerable plaques and minor lesions, such as intimal erosions and tears. A number of coronary angiograms may indicate borderline lesions with moderate stenosis. OCT examination may uncover the presence of unstable lesions with a high possibility of acute events. Therefore, the study of borderline lesions should shift the focus to screening for high-risk vulnerable plaques and ensure early intervention to reduce ACS events. The new generation of OCT integrates the detection function of FFR and obtains morphological and functional information at the same time. This achieves the guiding effect of optimizing the treatment of critical lesions $(28,29)$.
OCT assists in guiding PCI treatment. For patients requiring PCI treatment, surgeons may use OCT to analyze the composition and characteristics of the plaque in order to guide the pre-treatment or pre-dilation of the diseased vessel and to help the interventionist select the length, size and type of the stent or balloon.

Selection of the stent diameter. The principle of selecting the diameter of the stent to guide CAG is that the ratio of the diameter of the stent to the vessel diameter is 1.1:1. However, only the size of the stent is selected based on the results of the angiography and the opinions of different surgeons may vary. Since OCT may display the exact blood vessel contour and vascular wall structure, the selection of the diameter of the stent is more accurate compared with CAG (28). In addition, OCT may indicate the reference blood vessel diameter according to the severity and stability of the vascular disease at both ends of the lesion. The ILUMIEN III study selected different stent schemes according to whether the media may be detected in the reference segment of the OCT image (26). If the media are visible, the minor media layer diameter (usually the distal end) is measured as the reference value of the stent 

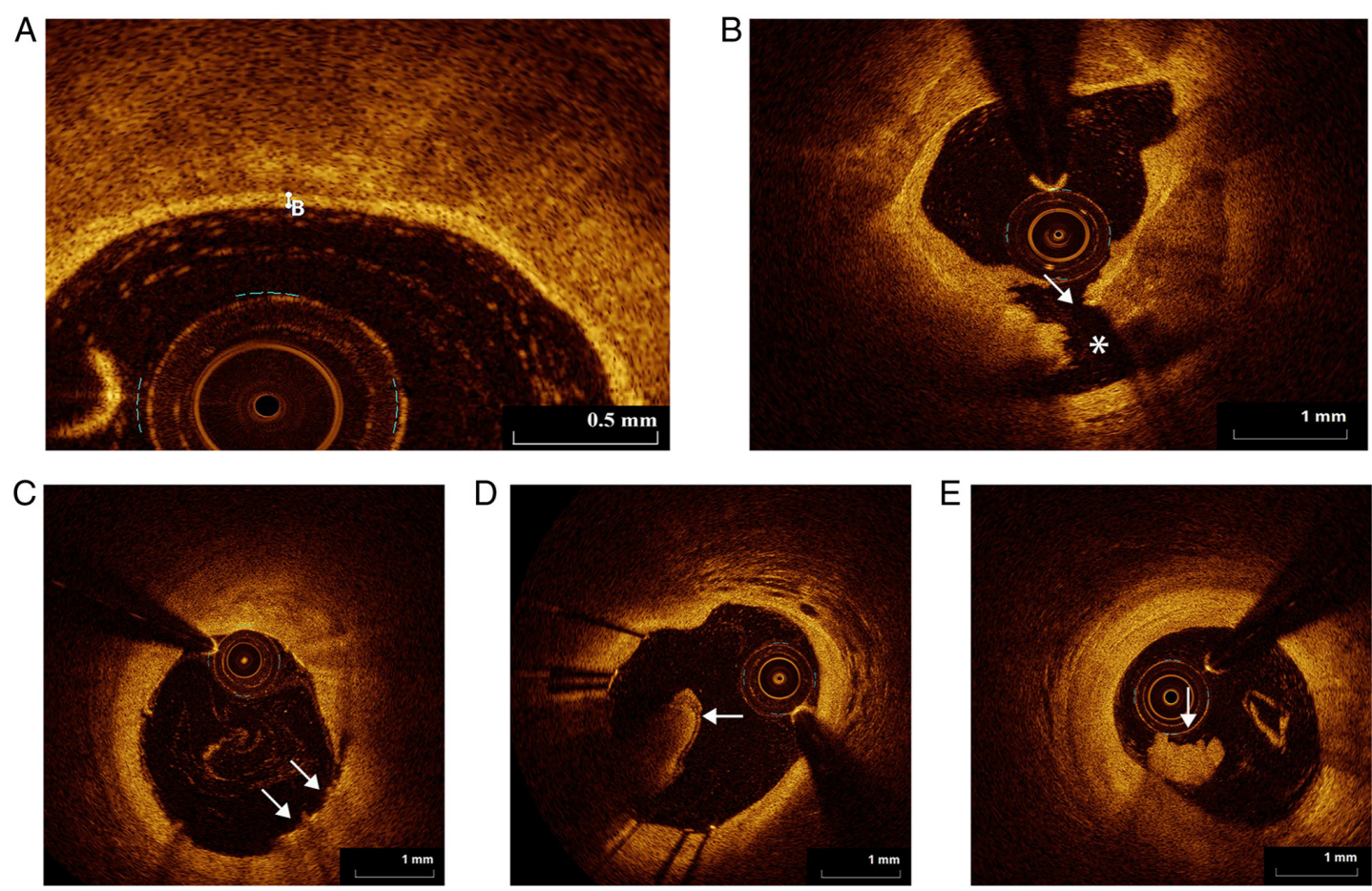

Figure 2. Vulnerable plaques. (A) Thin-cap fibroatheroma: The thinnest fiber cap ( $40 \mu \mathrm{m}$, point B; scale bar, $0.5 \mathrm{~mm}$ ). (B) The plaque is ruptured, the continuity of the fiber cap is interrupted (arrow) and a cavity is formed (asterisk); (C) plaque erosion and complete fibrous cap continuity with mural thrombus (arrow); (D) red thrombus (arrow); (E) white thrombus (arrow; scale bars, $1 \mathrm{~mm}$ ).

diameter. If the media is not visible, the minimum diameter of the reference segment automatically measured by the machine is selected as the reference value of the stent diameter. In the era of bioabsorbable stents, in theory, it may be more appropriate to select the diameter of the stent based on the media to prevent the thicker surface of the stent rod from protruding into the lumen and affecting blood flow (30).

Determination of the requirement for stent post-expansion. The requirement for post-expansion following stent implantation is mainly determined by the attachment and expansion of the stent. In principle, the expansion rate of the metal stent should reach $>80 \%$ and the expansion rate of the absorbable stent should reach $>90 \%$. Poor stent expansion is closely associated with stent restenosis and thrombosis in the stent. The ILUMIEN II study confirmed that OCT and IVUS have comparable efficacies in evaluating stent expansion $(30,31)$. If the stent displays poor expansion, a non-compliant balloon of the same caliber should be used to apply high pressure and fully expand the stent. The determination of poor stent adhesion by using OCT is more sensitive compared with IVUS. In the OCT image, the 'expansion rate' may be used to evaluate stent expansion. In general, the expansion rate is $90 \%$ critical for evaluating the immediate underexpansion. The ILUMIEN I study determined that the OCT detection underexpansion ratio may be as high as $41.3 \%$ (28). The stent expansion rate is calculated as follows:

$$
\text { Stent expansion rate }=\frac{\text { minimum stent area }}{\text { average reference vessel segment area }} \times 100 \%
$$

Selection of the landing point of the support. IVUS and OCT studies have indicated that, if the stent is placed on an unstable plaque, it is prone to long-term restenosis or edge dissection after implantation (30-32). Of note, in theory, the stent should be placed on the regular blood vessel segment, where angiography appears normal, OCT and IVUS examinations still have specific load plaques (even TCFA), so OCT examinations should be used and the selection is relatively stable. The normal blood vessel segment is used as a reference to accurately measure the diameter of the blood vessel, which not only ensures the efficacy of stent implantation but also avoids excessive damage to the blood vessel wall at the edges of the stent.

OCT evaluation of the immediate effect of stent PCI. The optimal stent implantation effect is that the stent adheres to the wall and expands well. Furthermore, it is not accompanied by dissection of the stent edge, tissue prolapse or thrombosis in the stent. OCT may identify a higher rate of complications after stent implantation, such as stent edge dissection, tissue prolapse and stent thrombosis.

Stent expansion. In the drug-eluting stent (DES) era, poor stent expansion is one of the fundamental reasons for in-stent restenosis and thrombosis. A previous study indicated that, whether it is a first-generation or second-generation drug stent, the minimum stent area (MSA) is $>5.0 \mathrm{~mm}^{2}$ (28). If the stent does not expand properly, the incidence of thrombosis in the stent will increase significantly within 1 year (33). Particularly in cases with left main disease, the MSA goal is to achieve circumflex branch opening $>5.0 \mathrm{~mm}^{2}$, anterior descending branch opening $>6.0 \mathrm{~mm}^{2}$, left main trunk end $>7.0 \mathrm{~mm}^{2}$ and left main trunk body $>8.0 \mathrm{~mm}^{2}$ (33). In general, calcified lesions and plaques form on a background of heavier 

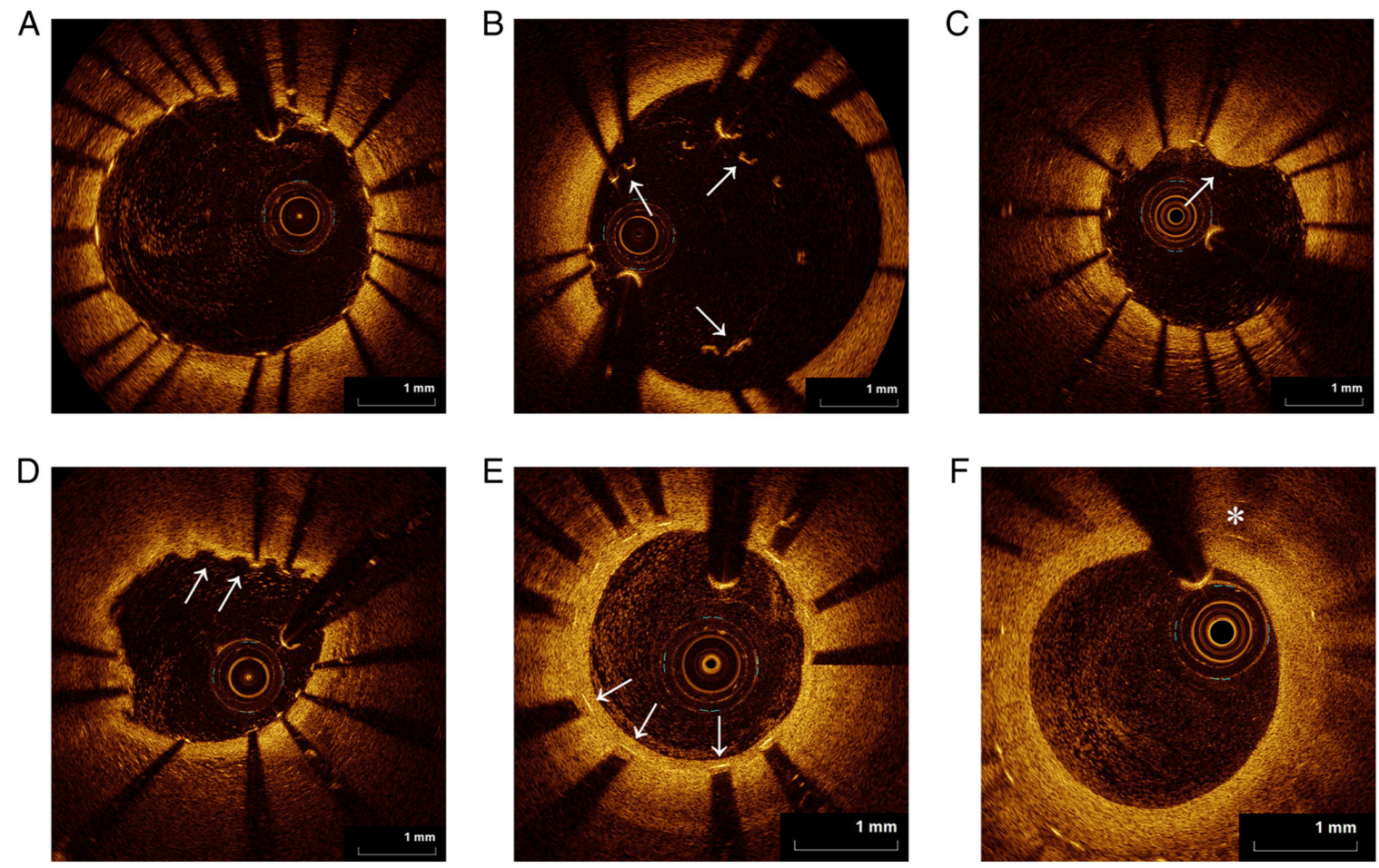

Figure 3. Evaluation after stent implantation. (A) The stent adheres well to the wall. (B) The stent is obviously poorly attached to the wall (indicated by the arrow). (C) Prolapse of the plaque (arrow). (D) Thrombus prolapse (arrow). (E) The stent trabeculae are embedded in the inner membrane and covered well (arrow). (F) Neoatherosclerosis in the stent. Hyperplastic endothelium within the stent is a high signal, followed by significant signal attenuation and blurred margins, suggesting lipid deposition, but not the formation of a true necrotic core (asterisk).

eccentric fibrous lesions, prone to poor stent expansion. Therefore, adequate pretreatment should be performed for such lesions. For instance, eccentric fibrous plaques should be fully pre-dilated and severely calcified lesions should be treated with rotational atherectomy or balloon incision (34). After PCI, poor stent expansion should be actively treated. Non-compliant balloons may be used to expand the stent by applying high pressure and OCT should be performed again after expansion to determine the adequacy of expansion.

Stent malapposition (SM). SM on OCT images means that the longitudinal distance from the stent trabecular surface to the lumen surface is greater than the thickness of the stent trabecula (if there is a polymer on the stent trabecula, it should also be included in the measurement). When the distance between the two is $>200 \mu \mathrm{m}$, this is defined as significant adhesion failure (Fig. 3A and B). Clinically, if the stent area poorly adhered to the wall is small and is not accompanied by poor stent expansion, no treatment is required (34). However, in the presence of $\mathrm{SM}$ and poor stent expansion, MSA $<5.0 \mathrm{~mm}^{2}$ post-expansion is recommended to ensure adequate stent expansion and adherence. In a follow-up study of OCT, poorly adhered stent filaments may also gradually be covered by endothelial cells and completely fuse with the vessel wall (35). Previous studies have performed OCT serial detection on 78 blood vessel segments where the stent wire was poorly attached immediately after surgery. The 6-month follow-up after the operation suggested that the volume of poorly adhered stents immediately after the operation decreased significantly and $75 \%$ of the stents that were poorly adhered immediately after the operation were completely fused with the vessel wall. This indicates that SM may delay the healing process of damaged blood vessels. When the distance between the stent wire and the vessel wall is narrow, the proliferating neointimal tissue may frequently cover the distance to the poorly adhered stent wire $(35,36)$.

Dissection of the stent edge and tissue prolapse. Following stent implantation, damage to the blood vessel wall often occurs at the edge of the stent. OCT is able to detect $37.8 \%$ of stent edge dissections (37). The edge dissection of the stent may be divided into intimal tear and media dissection. Intimal tear refers to the sheet-like lifting of the intima after stent implantation, without any obvious rupture of the plaque fibrous cap. Media dissection refers to the intimal tear extending to the media of the coronary artery, which may lead to intracoronary hematoma. In general, the severity of interlayer dissection, the angle at which the interlayer occurs and the length measured on the vertical axis images are taken into consideration. Stent edge dissection is closely associated with in-stent thrombosis and poor prognosis. Compared with superficial layer dissection, stent edge dissection involving the deep layer of the coronary artery wall significantly reduces the survival rate of patients without clinical events (38).

On OCT imaging, tissue prolapse is defined as tissue protruding from the lumen between the trabeculae of the stent after the stent is implanted. Tissue prolapse may be divided into plaque prolapse (Fig. 3C) and thrombosis (Fig. 3D). Plaque prolapse usually has a smooth surface 
Table VI. Treatment principles for vascular dissection after stent implantation and the main treatment principles for tissue prolapse in the stent after stent implantation.

A, Principles of treatment of vascular dissection after stent implantation

Type of vascular dissection

Processing principle

For intimal dissection with no clinical symptoms, no No special treatment

ischemic ECG changes, TIMI blood flow grade III

For dissection involving the vascular media, even Implant the stent immediately

intra-vascular hematoma or vascular rupture

The severity lies between the above two

If the edge of the stent dissection is $>60^{\circ}$, the length of the dissection is $>3 \mathrm{~mm}$, the distal TIMI blood flow is affected, and the MLA is $<5.0 \mathrm{~mm}^{2}$, the stent should be further implanted to avoid serious clinical

consequences

B, Major treatment principles of tissue prolapse in the stent after stent implantation

Type of tissue prolapse

Processing principle

The amount of tissue prolapse in the stent is small, the protruding lumen is $<200 \mu \mathrm{m}$, prolapsed area is $<10 \%$ of the area in the stent, the stent expands well and the TIMI blood flow is grade III

The amount of tissue prolapse in the stent is large, the protruding lumen $>200 \mu \mathrm{m}$, the prolapsed area is $\geq 10 \%$ of the internal area of the stent and the stent is poorly expanded

The effect of the above two methods is still not obvious

Should not intervene temporarily, postoperative antiplatelet therapy should be strengthened

Use of a balloon with the same diameter as the stent for high-pressure expansion

It may be considered to implant a stent in the prolapsed tissue to cover the prolapsed tissue to increase the effective lumen area

TIMI, thrombolysis in myocardial infarction; MLA, minimal luminal area.

without apparent signal attenuation, while thrombosis usually has an irregular surface with solid attenuation. The incidence of tissue prolapse after stent implantation is high. It was reported that $95 \%$ of stents may be detected by OCT immediately after implantation (30). The occurrence of tissue prolapse after PCI is associated with the nature of the plaque. When the stent is located on the TCFA or necrotic nucleus defined by OCT, tissue prolapse is likely to occur (39). Irregular tissue prolapse is an independent predictor of adverse clinical events after PCI (Table VI).

Immediate stent thrombosis. Immediate stent thrombosis on OCT appears as an irregular mass that protrudes into the lumen immediately after stent implantation. Although stent thrombosis is rare, it frequently leads to PCI complications with severe clinical events. At present, OCT is the only imaging technique other than angioscopy that may identify $\sim 100 \%$ of thrombi. For stent thrombosis detected by OCT, corresponding treatment measures should be taken according to the severity, the patient's clinical manifestations and the hemodynamic impact. First, for immediate stent thrombosis with low thrombus volume, no clinical symptoms, no ischemic ECG changes and no severe hemodynamic disorders, anticoagulation and dual antiplatelet therapy should be administered. If the volume of the thrombus in the stent is larger, the patient has obvious ischemic symptoms and severe hemodynamic disorders, a suction catheter may be used for thrombus aspiration to reduce the load of the thrombus in the stent. If there is no apparent improvement after aspiration, following intracoronary administration of a dose of tirofiban, a balloon with the same diameter as the stent should be used for low-pressure expansion. If the effect of the aforementioned method is still not sufficient, re-implanting a stent at the thrombus should be considered. The immediate causes of thrombosis in the stent (such as poor expansion, immediate SM and dissection of the stent edge) identified on OCT should be dealt with accordingly as described above (40).

\section{OCT guides complex lesion management}

Left main disease. Left main coronary artery lesions refer to lesions with stenosis of the left main coronary artery by $\geq 50 \%$ on CAG. This is one of the common clinically complex lesions. In recent years, with the advances in technology, left main bifurcation lesions are no longer considered to be a contraindication for PCI treatment (Table VII) $(27,41)$. 
Table VII. Several features used in the examination of left main lesions $(4,27,41)$

\section{$\mathrm{A}, \mathrm{CAG}$}

Consideration

Judement of the degree of stenosis

Diffuse left main disease

Left main ostium imaging

Left main artery

emergence

Coronary angiography

\section{Comments}

Surgeons have different judgments on the degree of stenosis of coronary angiography and they frequently underestimate the degree of stenosis of coronary artery disease

When the left main disease is diffuse, it is difficult for angiography to accurately reflect the diameter of the blood vessel, which affects the choice of stent diameter and ultimately affects the effect of PCI

During the left main ostium imaging, the contrast agent flows back to the aorta, resulting in unclear visualization of the coronary ostium

When the left main artery emerges from the aorta with an acute angle or the left main body is curved, the severe stenosis is easily missed by coronary angiography

Coronary angiography is not able to provide any detailed information on plaque composition and immediate effects after PCI

\section{$\mathrm{B}$, IVUS}

Consideration

Preoperative IVUS

examination

Intraoperative IVUS

Postoperative IVUS

IVUS in combination with FFR

\section{Comments}

Preoperative IVUS examination may confirm stenosis of the left main lesion, the length of the lesion and the characteristics of the plaque

Intraoperative IVUS may determine the position and accuracy of the reset side guidewire, exclude the guidewire from passing outside the stent and avoid serious deformation of the stent Postoperative IVUS examination may clarify the immediate effect of the stent and postoperative complications, and reduce the occurrence of postoperative cardiovascular adverse events IVUS combined with FFR may guide the choice of treatment strategies for borderline left main lesions

\section{C, OCT}

\section{Consideration}

OCT scanning speed

Accuracy of area and diameter measurement OCT evaluation of effects and OCT sensitivity ILUMIEN $^{\text {TM }}$ OPTIS ${ }^{\text {TM }}$ system real-time three-dimensional imaging

\section{ILUMIEN $^{\mathrm{TM}}$ OPTIS $^{\mathrm{TM}}$} system integration of FFR

OCT transmission depth

\section{Comments}

The scanning speed of the left main disease is faster $(25 \mathrm{~mm} / \mathrm{sec})$, which may reduce the inter ference of the cardiac cycle on coronary artery imaging Measurement of the smallest lumen area and diameter and the evaluation of the composition are more accurate (4)

OCT may clearly evaluate the immediate effects, vascular wall damage and complications after PCI, and is more sensitive than IVUS

The ILUMIEN ${ }^{\mathrm{TM}}$ OPTIS ${ }^{\mathrm{TM}}$ system may perform real-time three-dimensional imaging of the left main lesion, which may finely determine the extent, length, stenosis and reference blood vessel diameter of the lesion, and it is convenient for the surgeon to observe and analyze the blood vessel from multiple angles to make a correct assessment

The ILUMIEN $^{\mathrm{TM}}$ OPTIS ${ }^{\mathrm{TM}}$ system integrates the FFR function, so that it has both morphological and functional evaluation functions, so as to more comprehensively evaluate the coronary artery function

OCT transmission depth is small and the remodeling changes of the blood vessels and the surrounding conditions of the blood vessels cannot be evaluated. This is the drawback in guiding PCI treatment of the left main disease

OCT, optical coherence tomography; CAG, coronary angiography; IVUS, intravascular ultrasound; FFR, fractional flow reserve; PCI, percutaneous coronary intervention.

Bifurcation lesions. A bifurcation lesion of the coronary artery refers to a $>50 \%$ stenosis of the opening of the main vessel and branch vessel. This is a complex type of lesion with a high failure rate of coronary stent implantation (42). Atherosclerosis 
readily develops at the bifurcation of the blood vessels due to the high shear stress of blood flow.

Preoperative OCT examination may accurately measure the stenosis of the main branch and branch opening, the length of the lesion and the distribution and the nature of the plaque, and it may help the surgeon select the appropriate interventional device and branch stent treatment strategy. It should be determined whether single-stent or multi-stent technology may be used or whether branch vessels require to be protected. OCT indicates that, for the proximal wall of the branch in which TCFA frequently occurs, the double-stent technology (such as crush T-stenting) is required. The multi-layer stent trabecular attachment site may easily lead to poor stent adhesion, uneven drug distribution and vulnerable plaques. Rupture of the stent may lead to embolism of the distal branch and the occurrence of the 'no-flow' phenomenon (43). In terms of imaging, it may prevent the implantation of the stent on the TCFA. OCT may be used to guide the branch guidewire to enter the branch from the middle or distal mesh to achieve a satisfactory balloon anastomosis and expansion of the stent to adhere to the vascular wall.

OCT examination after surgery may be performed to observe the adhesion and endothelialization of the stent at the bifurcation, thereby reducing the occurrence of thrombosis in the stent. In addition, the real-time 3D imaging function of the ILUMIEN ${ }^{\text {TM }}$ OPTIS $^{\text {TM }}$ OCT system may also reveal the spatial distribution and structure of blood vessels, analyze the blood vessels at all angles freely in space and guide the re-entry of the guidewire (44). It was reported that $3 \mathrm{D}$-OCT-guided stent implantation for bifurcation lesions is feasible and may reduce the occurrence of stent malapposition (45). Therefore, this application should be considered as a guidance in the clinical treatment of bifurcation lesions.

Chronic occlusive disease. In recent years, with the development of interventional devices, considerable progress has been made in the treatment of chronic total occlusion (CTO), but the success rate of CTO intervention remains relatively low and faces several challenges (46). In this complex disease, the information provided by OCT may affect successful completion of the operation.

There have been reports of in vitro experiments using the forward-looking OCT system to perform multi-axis imaging of occluded blood vessels to construct cross-sectional images of the affected vessels (47). OCT is able to distinguish between different levels of the occluded lumen and vessel wall, and it is possible to identify microchannels. This information may be used to guide the wire through the lesion. Once the guidewire has passed through the occluded segment, OCT may detect the plaque component that caused the occlusion.

In addition, an OCT study indicated that the intimal repair of CTO lesions is delayed after stent implantation; thus, OCT may help determine the time limit of dual antiplatelet therapy following stent implantation in CTO lesions (48). It should be pointed out that the currently available evidence on OCT as a method for guiding the treatment of CTO lesions is insufficient and further research is required.
Calcified lesions. When severe annular calcification is detected on OCT, direct implantation of stents should be avoided and pre-dilation or experimental expansion strategies should be considered first (49). Balloon cutting or coronary atherectomy may be used in short calcification rings. It should be noted that there is currently no clear cutoff value (calcification angle, length or thickness) that may be used as an indication for balloon incision or coronary atherectomy (50).

Calcified lesions limit the expansion of blood vessels and balloons; therefore, calcified lesions frequently lead to poor stent expansion (49). OCT may accurately assess the expansion of the stent, thereby guiding the selection of a suitable post-expansion balloon. It was indicated that the calcification angle and calcification area measured by OCT are negatively correlated with the MSA and the minimal stent diameter after DES expansion (49).

\section{Application of OCT in the post-stent implantation fol- low-up}

OCT has significant advantages in assessing the repair of blood vessels after stenting and the mechanisms underlying treatment failure.

OCT assessment of intimal coverage. The thickness of the stent intima measured by OCT has been indicated to be highly correlated with the thickness of the tissue $(r=0.85, \mathrm{P}<0.01)$, with excellent repeatability of the thickness measurement between individuals or the same intima (51). OCT has a sensitivity of $80 \%$ and a specificity of $95 \%$ for detecting incomplete stent intimal coverage. For stent neointima (Fig. 3E) with a thickness of $>100 \mu \mathrm{m}$, it may be divided into three categories according to OCT image characteristics: The first is characterized by homogeneity, high reflection and a relatively uniform signal, with no local attenuation signal. The second involves layered, centripetal, double-layer or multi-layer optical signals, the near lumen side usually has high-reflection signals and the far cavity side usually has low-reflection signals Finally, the third category is characterized by heterogeneity, with low reflection and an uneven signal, with local solid signal attenuation.

With the advancement of technology, OCT study hasdetermined that the second-generation everolimus-eluting stent (EES) achieves significantly faster intimal repair compared with the first-generation sirolimus-eluting stent (SES) and the surface endothelialization is relatively complete (52). This discovery has prompted several scholars to consider that the existing dual-antiplatelet therapy strategy may be changed. It is recommended that the current dual-antiplatelet therapy is shortened to 9 months and certain scholars even proposed to shorten it to 6 months (53). It was also reported that, in patients with EES implantation, the 6-month dual-antibody treatment is not inferior to the 12-month treatment plan (54). The application of OCT to evaluate direct intimal coverage appears to be highly promising for guiding the clinical standards for dual-antibody treatment after stenting. To date, certain scholars have proposed using OCT to guide individualized dual-antibody therapy and examined whether it is possible to use OCT to evaluate the trabecular coverage of the stent to adopt an individualized antiplatelet therapy plan, 
and whether the characteristics of intimal tissue observed on OCT may be used in individualized dual anti-platelet therapy and basic anti-atherosclerosis treatment programs (51). However, due to the lack of a specific evaluation model and a unified evaluation plan, these are issues worthy of further consideration. Since the incidence of in-stent thrombosis is inherently low, a large sample size is required by further clinical studies to confirm the advantages of OCT in guiding antiplatelet therapy, which is associated with major practical difficulties. However, with the increase in follow-up data of OCT stents, real-world registration studies may help solve this problem and even change the existing guidelines on the recommended duration of dual-antibody application after stenting.

In terms of identifying the potential clinical risks and intervention factors of the neointima, OCT is a powerful tool that has achieved significant research results. Kubo et al (55) reported that intimal stent coverage was significantly delayed in patients with UAP. Kochman et al (56) used OCT to follow up the DESs and bare metal stents (BMSs) of diabetic patients for 2 years and observed that the intima remained uncovered 2 years after the DES was implanted. Furthermore, the intimal coverage of the stent is unrelated to whether the patient has diabetes. A Japanese clinical study on OCT indicated that atorvastatin treatment may affect the migration of endothelial progenitor cells (EPCs), thereby accelerating the repair and endothelialization of the endothelium after SES implantation. In addition, different statin drugs may affect EPC migration (57). There are apparent differences in the promotion effect of membrane repair. These studies have provided research directions and intervention targets for promoting stent intimal healing and reducing long-term stent-related clinical problems from the perspective of OCT.

OCT assessment of neoatherosclerosis. It was initially suggested that, at 6-12 months after BMS implantation, neointimal hyperplasia caused lumen stenosis. As the tissue matures, the lumen area tends to stabilize or even improve (58). However, an increasing number of studies have confirmed that the stable fibrotic endometrial tissue in these stents may gradually develop into unstable atheromatous plaque tissues, leading to late stent failure (58-60). Complex atherosclerosis that occurs in the neointimal tissue after stent implantation is defined as neoatherosclerosis (Fig. 3F). Regarding the phenomenon of neoatherosclerosis plaques in stents, the underlying mechanisms and clinical intervention methods remain to be explored, which is an important direction for future basic and clinical research.

OCT may serve an important role in the mechanism and intervention of neoatherosclerosis. The neoatherosclerosis in DES is likely associated with severe local inflammation in the early stage after stent implantation. Furthermore, neoatherosclerosis is mainly distributed around the trabeculae. The occurrence of plaque in a bare stent is gradually formed after the stent intima proliferates to a certain thickness. The new plaque is at a certain distance from the trabecula of the stent, which is similar to the occurrence of coronary plaque in situ. An OCT study demonstrated that the progression of neointimal atherosclerosis after stent implantation is associated with the progression of an in situ plaque in the autologous coronary artery (60). This result is expected, as the new plaque has the same risk as the in situ plaque. In addition, similar to in situ plaques, new blood vessels have an essential role in the occurrence and development of new plaques in stents, particularly in diabetic patients $(61,62)$. An OCT study determined through multiple regression analysis that low-density lipoprotein-cholesterol levels $>70 \mathrm{mg} / \mathrm{dl}$, smoking and chronic kidney disease are risk factors that promote the occurrence of new plaques. By contrast, angiotensin-converting enzyme inhibitors are effective defense pathways that prevent the occurrence of new plaques (63). Therefore, this type of drug may inhibit the formation of neoatherosclerosis plaques to a certain extent. Traditional cardiovascular risk factors, such as sex, hypertension, diabetes and hyperlipidemia, cannot predict the occurrence of neoatherosclerosis plaques following stent implantation (64); this demonstrates that, although the histological and imaging characteristics of neoatherosclerotic plaques and primary atherosclerosis are similar, there may be marked differences in the underlying mechanisms. With the advancement of evaluation methods, early screening of neoatherosclerosis plaques and active control of related risk factors may help improve the long-term prognosis after PCI.

OCT determines the cause of stent failure. The main reasons for stent failure are in-stent restenosis (ISR), intra-stent thrombosis, $\mathrm{SM}$ and stent fracture (SF). However, it is challenging to discover the pathological mechanisms leading to the failure of stent implantation by angiography alone. The use of OCT to follow up patients with late stent implantation failure may help elucidate their types and underlying mechanisms, which is conducive to the improvement of stent design and the optimization of surgical procedures, thereby preventing stent failure caused by various mechanisms.

ISR. ISR may be divided into imaging and clinical restenosis. Following stent implantation, the former refers to the CAG performed to confirm that the stent implantation segment loses $\geq 50 \%$ of the lumen. On OCT images, ISR means that the area in the neointima of the stent exceeds $50 \%$ of the area of the stent. OCT may quantitatively measure ISR at the lesion level, mainly by measuring the thickness of the neointima, the area of the neointima, the calculated volume of the neointima and the volume of neointimal hyperplasia obstruction rate (the ratio of the average neointimal volume to the average stent volume) to assess the degree of ISR.

Previous studyreported that coronary artery balloon dilatation, paclitaxel drug-coated balloon or DES implantation may be performed for ISR (65). During a follow-up over 200 days, the histological characteristics of the stent intima detected by OCT were indicated to be associated with the reappearance of ISR and the prognosis of restenosis with different tissue characteristics was significantly different with different treatment methods. It is suggested that the causes of restenosis of clinical stents are different (intimal hyperplasia, atherosclerosis and stent thrombosis) and the treatment methods for restenosis should also be different. Therefore, OCT examination may help analyze the causes of ISR and the degree of stenosis and help guide treatment planning. 
Stent thrombosis. The detection sensitivity of OCT for stent thrombosis is higher compared with that of CAG and IVUS. OCT may identify thrombi and distinguish between red and white thrombi by the thrombus components, which is of significant reference value for assessing the composition of acute stent thrombosis. OCT may detect small thrombi attached between the stent wires that have not adhered to the wall and provide accurate measurement of the size and length of the stent and the luminal area of the blood vessel. In addition, OCT has a specific guiding effect on the dosage and course of clinical antithrombotic drugs (66).

Guagliumi et al (67) studied 18 patients with advanced stent thrombosis in DES. All patients underwent thrombus aspiration prior to OCT and IVUS examinations. It was determined that the non-filament coverage rate in the stent thrombosisgroup was $12.27 \%$, which was significantly higher compared with the $4.14 \%$ in the control group. The results of IVUS demonstrated that the stent expansion degree of the two groups was similar but the positive vascular remodeling was more significant in the advanced stent thrombosis group. Multivariate analysis suggested that the uncovered segment length of the stent wire determined by OCT and the IVUS remodeling index were independent predictors of advanced stent thrombosis (67).

Late SM (LSM). SM refers to the apparent separation of the stent trabeculae from the vessel wall and may be divided into early and late SM. A previous study demonstrated that LSM may cause stent thrombosis. Compared with the control group, the incidence of SM in patients with very advanced thrombosis ( 77 vs. $12 \%$, respectively; $\mathrm{P}<0.001)$ and the area of SM (77 vs. $12 \%$, respectively; $\mathrm{P}<0.001$ ) were significantly increased (68). A total of 20 cases of late and very late stent thrombosis were examined through OCT, indicating that 11 cases $(55 \%)$ had SM, of which 5 cases had LSM caused by abnormal positive remodeling of the vessel wall (68). However, the incidence of LSM is notably higher than the incidence of late stent thrombosis, indicating that, although LSM may not be the sole cause of stent thrombosis, it may be one of the critical factors contributing to the formation of stent thrombosis.

OCT carries unique advantages in assessing postoperative SM. The high resolution of OCT may detect SM not detected by IVUS. As IVUS cannot detect this type of SM, there is no corresponding balloon expansion. However, the ratio of SM detected by OCT is relatively high (69). When compared with IVUS, is it unclear whether the higher number of SMs detected by OCT require balloon expansion, whether these SMs may self-repair and what is their prognosis. These questions must be addressed through further clinical research.

$S F$. The incidence of SF is low. However, with the increase in the number of stent implantations, an increasing number of SF cases are being reported, particularly in cases of DES implantation (70). In the past, OCT machines lacked 3D imaging tools and OCT cross-sectional images were not associated with obvious advantages in SF. The emergence of OCT 3D real-time reconstruction systems enables the use of OCT to study the occurrence of SF and its clinical significance (30,71). 3D OCT may help diagnose SF and determine the effect of
SF on local endometrial repair and local external stent plaque. Through OCT, it is also possible to identify the factors that are likely to cause SF.

While, SF may cause local mechanical vascular irritation, leading to inflammation and neointimal hyperplasia, the fracture destroys the local stent structure, causing thrombosis and blood flow blockage and affecting the blood supply to the heart. Stent trabecular fracture may also represent a potential mechanism underlying ISR and stent thrombosis. The incidence of SF in EES has been reported to be $1.7 \%$. The 9-month follow-up indicated that SF was associated with revascularization of the target lesion caused by the event (18.7 vs. 2.3\%) (72). The incidence of fracture after implantation of the noboribiolimus-eluting stent reached $4.1 \%$, which is also associated with the revascularization of the target lesion induced by ischemic events (73).

\section{Factors and common problems affecting OCT imaging results}

OCT imaging results rely on standards and correct operational techniques. In order to optimize the quality of OCT images, the operator requires certain techniques to guide catheter operations. Several factors affect the results of OCT imaging, including excessive branch blood vessels, excessive lumen width, excessive vascular bending, severe stenosis of the lumen, the effect of cardiac cycle motion on the image and factors related to intraoperative procedures. During the operation, attention should be paid to the coaxiality of the guide tube and the target vessel, the use of a contrast agent bolus, the synchronization of image acquisition and the avoidance of red blood cells or air bubbles in the imaging catheter or in the bolus contrast agent.

Branch vessel. When encountering a relatively large branch vessel, it may affect the measurement accuracy and image recognition. At this time, it is necessary to omit the branch vessel segment and select a normal lumen to start a new measurement (Fig. 4A).

Bubble artifacts. The formation of small bubbles in the silicone lubricant between the sheath and the optical fiber may attenuate the blood vessel wall signal in the corresponding area. This type of image is not suitable for the analysis of tissue characteristics (Fig. 4B).

Saturation artifacts. The high specular reflection of the beam (usually the stent trabeculae) produces an amplified signal, which appears as axial linear stripes on the image (Fig. 4C).

Incomplete image. If the image area is $<3 / 4$ of the normal area, it is difficult to ensure the accuracy of the measurement data. In such cases, this frame or segment of the image may be omitted from the analysis (Fig. 4D).

Unwashed blood. When the blood in the lumen is not washed out during imaging, the red blood cells defocus the light beam and weaken the brightness of the vessel wall, creating an artifact. Furthermore, residual blood must be distinguished from a thrombus (Fig. 4E). 

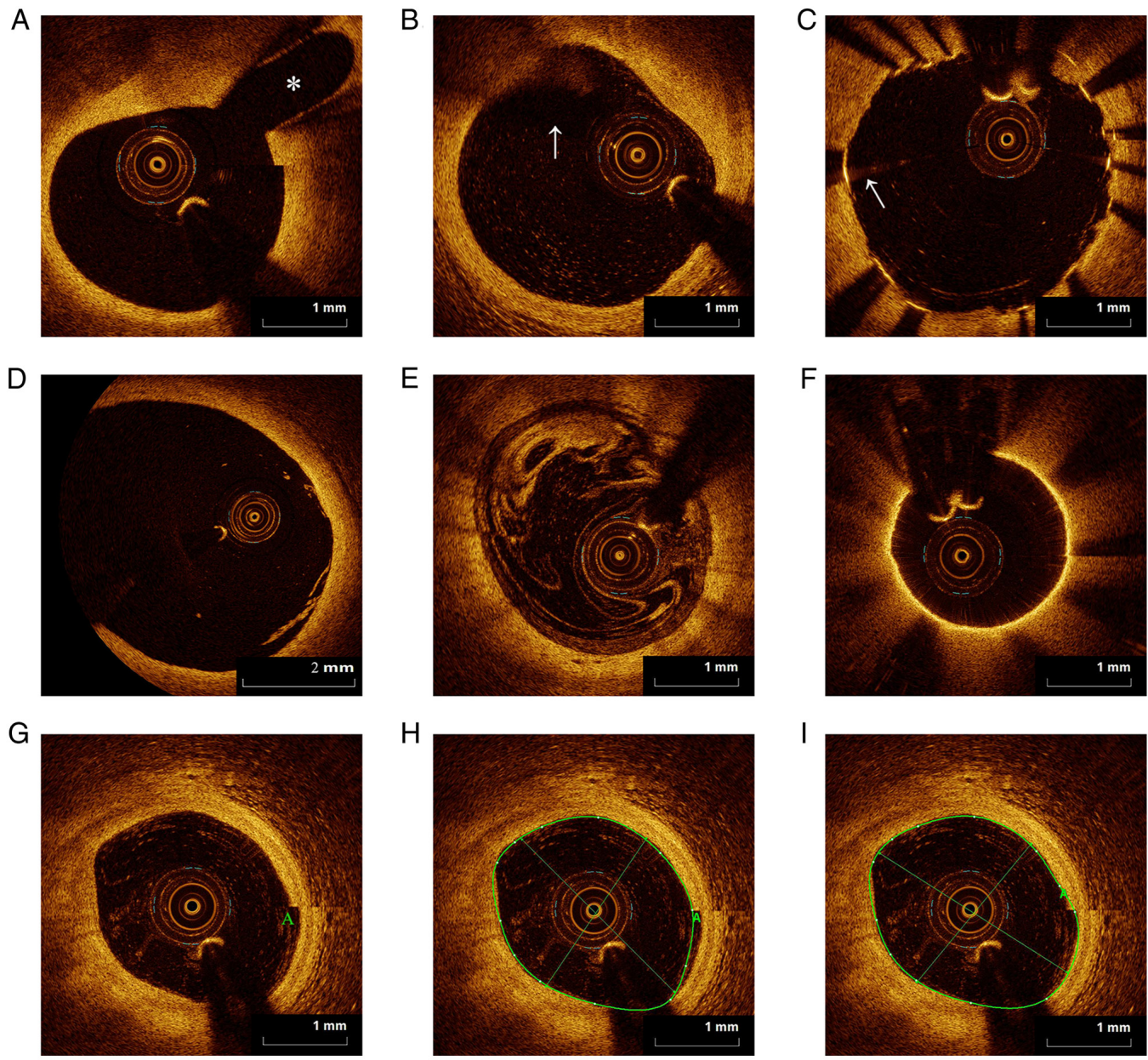

Figure 4. Factors affecting OCT imaging results. (A) Branch vessel (asterisk). (B) Bubble artifacts (arrow). (C) Saturation artifacts (arrow; scale bar, 1 mm). (D) Incomplete image (scale bar, $2 \mathrm{~mm}$ ). (E) Blood that was not washed away. (F) The guidewire is damaged. (G) Staggered layer artifacts. (H) The incorrect measurement method was used at the fault level. (I) The correct measurement method was used at the staggering level (scale bar, $1 \mathrm{~mm}$ ). The A in G-I indicates the staggering layer. The green line indicates the measured lumen profile.

Guidewire damage. When the guidewire is damaged, abnormal images may be obtained. Fig. 4F presents images occurring when the two guidewires are damaged.

Splitting artifacts. In one frame of imaging, the artery or the guidewire is quicker than the other, causing the signal points of the lumen boundary to be out of sync, resulting in layering artifacts. This must be distinguished from intimal tears (Fig. 4G and $\mathrm{H}$ ).

\section{Application prospects of OCT in the field of cardiovas- cular interventional diagnosis and treatment}

With the continuous improvement of OCT, it is expected to have an important role in the examination of coronary intravascular lesions, the optimization of PCI strategies and the development of novel stents. The expected future development directions and advantages of OCT are listed below (29,74-78).

i) The currently used frequency domain-OCT retracement speed is up to $40 \mathrm{~mm} / \mathrm{sec}, 158$ frames of images $/ \mathrm{sec}$, and the A-line frequency is $81 \mathrm{kHz}$. This causes $2-3$ heartbeats to be affected every $50 \mathrm{~mm}$ of retracement, resulting in blurred $3 \mathrm{D}$ imaging edges. If the withdrawal speed was to be accelerated to $>100 \mathrm{~mm} / \mathrm{sec},>3,000$ frames of images $/ \mathrm{sec}$ and A-line frequency $>1.5 \mathrm{MHz}$, and combined with catheter positioning and tracking technology, the 3D imaging would be clearer and more accurate, and the clinical guidance value would be significantly improved. The bolus of the contrast agent should also be markedly reduced.

ii) Micro-resolution OCT (micro-OCT) has a resolution of up to $1 \mu \mathrm{m}$, which may analyze the microstructure of the atherosclerotic plaque, plaque rupture, thrombus, neointima and other tissues, at the cellular or subcellular level. The cellular components of the plaque may provide a novel imaging method. Once micro-OCT is used in human research, it may dynamically observe certain cellular components and the stability and correlation of atherosclerotic plaques or determine which cellular components (such as endothelial cells, macrophages and smooth muscle cells) are able to affect the intima of the stent. These future applications of OCT may lead to breakthroughs in the current understanding of the development of atherosclerosis and stent intimal coverage, and may change existing views and provide novel therapeutic intervention targets.

iii) Combining two or more imaging technologies may be used to make up for the deficiencies of a single imaging method. OCT-IVUS: Integrating OCT and IVUS on one imaging catheter not only takes advantage of the high resolution of OCT, but also penetrates through the IVUS catheter, 
and the depth is markedly increased. OCT-NIRS: NIRS may perform an accurate and real-time in vivo assessment of lipid core plaques. Combining NIRS technology with OCT and applying them to intracoronary imaging may not only uncover the morphological characteristics of the plaques but may also identify the chemical composition of the plaque.

iv) Real-time integration of structural and functional imaging information, such as OCT-FFR institutional functional testing technology, may provide more accurate assessment and guidance of treatment strategies for critical lesions, while improving the current status of excessive implantation of stents in the clinical setting.

v) Combining OCT and fluorescent molecular labeling technology may provide information on inflammation and endothelial function in the plaque, which is conducive to the in-depth study of the pathophysiological processes of the atherosclerotic plaque and the processes involved in stent repair.

vi) More large-sample OCT prospective clinical studies are required to change or improve existing interventional treatment strategies in certain controversial areas. Accumulating the clinical data of OCT in determining the characteristics of lesions and guiding precision interventional treatment may promote the priority recommendation level of OCT in guidelines for the diagnosis and treatment or revascularization in coronary heart disease.

\section{Summary}

OCT is a high-resolution intravascular imaging method for evaluating the composition of the coronary arterial wall and the stability of the atherosclerotic plaque, optimizing the efficacy of stent implantation, and evaluating the long-term effectiveness and safety of the stent. Currently, the interventional diagnosis and treatment of coronary heart disease require better interventional results for patients, as well as individualized and customized optimal strategies. With the innovation and progress of OCT technology, clinical research evidence is markedly more sufficient and interventional physicians have mastered the OCT operating system. It is thought that the application of this modality in clinical and basic research of coronary artery atherosclerosis, the selection of treatment strategies for acute coronary syndromes, the optimization of interventional treatment effects, the evaluation of the effect of novel stents, the stent intima coverage and the selection of double antibodies, have become increasingly extensive. Furthermore, to a certain extent, OCT may change or affect the currently applied coronary interventional treatment strategies $(4,5,79)$.

\section{Acknowledgements}

Not applicable.

\section{Funding}

This paper was supported by the Korea Food Research Institute funded project (grant no. 2017029 to QZ) and the National Natural Science Foundation of China (grant no. 61671098 to QZ).

\section{Availability of data and materials}

Data sharing is not applicable to this article, as no datasets were generated or analyzed during the current study.

\section{Authors' contributions}

JW and SY wrote and revised the manuscript. JQ edited the manuscript. QZ performed the literature review. ZJ designed and conceived the the study. All authors have read and approved the final manuscript. Data authentication is not applicable.

\section{Ethics approval and consent to participate}

Not applicable.

\section{Patient consent for publication}

Not applicable.

\section{Competing interests}

The authors declare that they have no competing interests.

\section{References}

1. Ramasamy A, Chen Y, Zanchin T, Jones DA, Rathod K, Jin C, Onuma Y, Zhang YJ, Amersey R, Westwood M, et al: Optical coherence tomography enables more accurate detection of functionally significant intermediate non-left main coronary artery stenoses than intravascular ultrasound: A meta-analysis of 6919 patients and 7537 lesions. Int J Cardiol 301: 226-234, 2020.

2. Bouma BE, Villiger M, Otsuka K and Oh WY: Intravascular optical coherence tomography [Invited]. Biomed Opt Express 8: 2660-2686, 2017.

3. van der Sijde JN, Karanasos A, van Ditzhuijzen NS, Okamura T, van Geuns RJ, Valgimigli M, Ligthart JM, Witberg KT, Wemelsfelder S, Fam JM, et al: Safety of optical coherence tomography in daily practice: A comparison with intravascular ultrasound. Eur Heart J Cardiovasc Imaging 18: 467-474, 2017

4. Tearney GJ, Regar E, Akasaka T, Adriaenssens T, Barlis P, Bezerra HG, Bouma B, Bruining N, Cho JM, Chowdhary S, et al: Consensus standards for acquisition, measurement, and reporting of intravascular optical coherence tomography studies: A report from the international working group for intravascular optical coherence tomography standardization and validation. J Am Coll Cardiol 59: 1058-1072, 2012.

5. Prati F, Regar E, Mintz GS, Arbustini E, Di Mario C, Jang IK, Akasaka T, Costa M, Guagliumi G, Grube E, et al: Expert review document on methodology, terminology, and clinical applications of optical coherence tomography: Physical principles, methodology of image acquisition, and clinical application for assessment of coronary arteries and atherosclerosis. Eur Heart J 31: 401-415, 2010.

6. Prati F, Guagliumi G, Mintz GS, Costa M, Regar E, Akasaka T, Barlis P, Tearney GJ, Jang IK, Arbustini E, et al: Expert review document part 2: Methodology, terminology and clinical applications of optical coherence tomography for the assessment of interventional procedures. Eur Heart J 33: 2513-2520, 2012.

7. Jang IK, Tearney GJ, MacNeill B, Takano M, Moselewski F, Iftima N, Shishkov M, Houser S, Aretz HT, Halpern EF and Bouma BE: In vivo characterization of coronary atherosclerotic plaque by use of optical coherence tomography. Circulation 111: 1551-1555, 2005.

8. Muller JE, Tofler GH and Stone PH: Circadian variation and triggers of onset of acute cardiovascular disease. Circulation 79: 733-743, 1989. 
9. Kolodgie FD, Burke AP, Farb A, Gold HK, Yuan J, Narula J, Finn AV and Virmani R: The thin-cap fibroatheroma: A type of vulnerable plaque: The major precursor lesion to acute coronary syndromes. Curr Opin Cardiol 16: 285-292, 2001.

10. Naghavi M, Libby P, Falk E, Casscells SW, Litovsky S, Rumberger J, Badimon JJ, Stefanadis C, Moreno P, Pasterkamp G, et al: From vulnerable plaque to vulnerable patient: A call for new definitions and risk assessment strategies: Part II. Circulation 108: 1772-1778, 2003.

11. Lin P, Ji HH, Li YJ and Guo SD: Macrophage plasticity and atherosclerosis therapy. Front Mol Biosci 8: 679797, 2021.

12. Nagasawa A, Otake H, Kawamori H, Toba T, Sugizaki Y, Takeshige R, Nakano S, Tanimura K, Takahashi Y, Fukuyama Y, et al: Relationship among clinical characteristics, morphological culprit plaque features, and long-term prognosis in patients with acute coronary syndrome. Int $\mathrm{J}$ Cardiovasc Imaging 37: 2827-2837, 2021

13. Kanaya T, Noguchi T, Otsuka F, Asaumi Y, Kataoka Y, Morita Y, Miura H, Nakao K, Fujino M, Kawasaki T, et al: Optical coherence tomography-verified morphological correlates of high-intensity coronary plaques on non-contrast T1-weighted magnetic resonance imaging in patients with stable coronary artery disease. Eur Heart J Cardiovasc Imaging 20: 75-83, 2019.

14. Ali ZA, Karimi Galougahi K, Mintz GS, Maehara A, Shlofmitz RA and Mattesini A: Intracoronary optical coherence tomography: State of the art and future directions. EuroIntervention 17: e105-e123, 2021.

15. Chang K, Ahn Y, Lim S, Yang JH, Lee KY, Choo EH, Kim HK Nam CW, Kim W, Hwang JY, et al: 2021 Korean society of myocardial infarction expert consensus document on revascularization for acute myocardial infarction. Korean Circ J 51: 289-307, 2021.

16. Sun R, Qiao Y, Yan G, Wang D, Zuo W, Ji Z, Zhang X, Yao Y, Ma G and Tang C: Association between serum adipsin and plaque vulnerability determined by optical coherence tomography in patients with coronary artery disease. J Thorac Dis 13 : 2414-2425, 2021

17. Milzi A, Lemma ED, Dettori R, Burgmaier K, Marx N, Reith S and Burgmaier M: Coronary plaque composition influences biomechanical stress and predicts plaque rupture in a morpho-mechanic OCT analysis. Elife 10: e64020, 2021.

18. Shi X, Gao J, Lv Q, Cai H, Wang F, Ye R and Liu X: Calcification in atherosclerotic plaque vulnerability: Friend or foe? Front Physiol 11: 56, 2020

19. Joo SP, Lee SW, Cho YH, Kim YS, Seo BR, Kim HS and Kim TS Vasa vasorum densities in human carotid atherosclerosis is associated with plaque development and vulnerability. J Korean Neurosurg Soc 63: 178-187, 2020.

20. Nishida T, Hiro T, Takayama T, Sudo M, Haruta H, Fukamachi D, Hirayama A and Okumura Y: Clinical significance of microvessels detected by in vivo optical coherence tomography within human atherosclerotic coronary arterial intima: A study with multimodality intravascular imagings. Heart Vessels 36: 756-765, 2021.

21. Leistner DM, Kränkel N, Meteva D, Abdelwahed YS, Seppelt C, Stähli BE, Rai H, Skurk C, Lauten A, Mochmann HC, et al Differential immunological signature at the culprit site distinguishes acute coronary syndrome with intact from acute coronary syndrome with ruptured fibrous cap: Results from the prospective translational OPTICO-ACS study. Eur Heart J 41 3549-3560, 2020.

22. Crea F and Libby P: Acute coronary syndromes: The way forward from mechanisms to precision treatment. Circulation 136 : $1155-1166,2017$

23. Wang J, Fang C, Zhang S, Li L, Lu J, Wang Y, Wang Y, Yu H, Wei G, Yin Y, et al: Systemic and local factors associated with reduced thrombolysis in myocardial infarction flow in ST-segment elevation myocardial infarction patients with plaque erosion detected by intravascular optical coherence tomography. Int J Cardiovasc Imaging 37: 399-409, 2021.

24. Kataoka Y, Puri R, Hammadah M, Duggal B, Uno K, Kapadia SR Tuzcu EM, Nissen SE and Nicholls SJ: Spotty calcification and plaque vulnerability in vivo: Frequency-domain optical coherence tomography analysis. Cardiovasc Diagn Ther 4: 460-469, 2014.

25. Vink A, Schoneveld AH, Richard W, de Kleijn DP, Falk E, Borst $\mathrm{C}$ and Pasterkamp G: Plaque burden, arterial remodeling and plaque vulnerability: Determined by systemic factors? J Am Coll Cardiol 38: 718-723, 2001.
26. Usui E, Yonetsu T, Kanaji Y, Hoshino M, Yamaguchi M, Hada M, Hamaya R, Kanno Y, Murai T, Lee T and Kakuta T: Efficacy of optical coherence tomography-derived morphometric assessment in predicting the physiological significance of coronary stenosis: Head-to-head comparison with intravascular ultrasound. EuroIntervention 13: e2210-e2218, 2018.

27. de la Torre Hernandez JM, Baz Alonso JA, Gómez Hospital JA, Alfonso Manterola F, Garcia Camarero T, Gimeno de Carlos F, Roura Ferrer G, Recalde AS, Martínez-Luengas IL, Gomez Lara J, et al: Clinical impact of intravascular ultrasound guidance in drug-eluting stent implantation for unprotected left main coronary disease: Pooled analysis at the patient-level of 4 registries. JACC Cardiovasc Interv 7: 244-254, 2014.

28. Wijns W, Shite J, Jones MR, Lee SW, Price MJ, Fabbiocchi F, Barbato E, Akasaka T, Bezerra H and Holmes D: Optical coherence tomography imaging during percutaneous coronary intervention impacts physician decision-making: ILUMIEN I study. Eur Heart J 36: 3346-3355, 2015.

29. Kedhi E, Berta B, Roleder T, Hermanides RS, Fabris E, IJsselmuiden AJJ, Kauer F, Alfonso F, von Birgelen C, Escaned J, et al: Thin-cap fibroatheroma predicts clinical events in diabetic patients with normal fractional flow reserve: The COMBINE OCT-FFR trial. Eur Heart J 42: 4671-4679, 2021

30. Lee $\mathrm{CH}$ and Hur SH: Optimization of percutaneous coronary intervention using optical coherence tomography. Korean Circ J 49: 771-793, 2019.

31. Maehara A, Ben-Yehuda O, Ali Z, Wijns W, Bezerra HG, Shite J, Généreux P, Nichols M, Jenkins P, Witzenbichler B, et al: Comparison of stent expansion guided by optical coherence tomography versus intravascular ultrasound: The ILUMIEN II study [observational study of optical coherence tomography (OCT) in patients undergoing fractional flow reserve (FFR) and percutaneous coronary intervention]. JACC Cardiovase Interv 8: 1704-1714, 2015.

32. Imola F, Mallus MT, Ramazzotti V, Manzoli A, Pappalardo A, Di Giorgio A, Albertucci M and Prati F: Safety and feasibility of frequency domain optical coherence tomography to guide decision making in percutaneous coronary intervention. EuroIntervention 6: 575-581,2010.

33. Bangalore S, Barsness GW, Dangas GD, Kern MJ, Rao SV, Shore-Lesserson L and Tamis-Holland JE: Evidence-based practices in the cardiac catheterization laboratory: A scientific statement from the american heart association. Circulation 144: e107-e119, 2021.

34. Viceconte N, Chan PH, Barrero EA, Ghilencea L, Lindsay A, Foin N and Di Mario C: Frequency domain optical coherence tomography for guidance of coronary stenting. Int J Cardiol 166: 722-728, 2013.

35. Räber L, Mintz GS, Koskinas KC, Johnson TW, Holm NR, Onuma Y, Radu MD, Joner M, Yu B, Jia H, et al: Clinical use of intracoronary imaging. Part 1: Guidance and optimization of coronary interventions. An expert consensus document of the European association of percutaneous cardiovascular interventions. Eur Heart J 39: 3281-3300, 2018.

36. Im E, Hong SJ, Ahn CM, Kim JS, Kim BK, Ko YG, Choi D, Jang Y and Hong MK: Long-term clinical outcomes of late stent malapposition detected by optical coherence tomography after drug-eluting stent implantation. J Am Heart Assoc 8: e011817, 2019.

37. Kume T and Uemura S: Current clinical applications of coronary optical coherence tomography. Cardiovasc Interv Ther 33: 1-10, 2018.

38. Onuma Y, Katagiri Y, Burzotta F, Holm NR, Amabile N, Okamura T, Mintz GS, Darremont O, Lassen JF, Lefèvre T, et al: Joint consensus on the use of OCT in coronary bifurcation lesions by the European and Japanese bifurcation clubs. EuroIntervention 14: e1568-e1577, 2019.

39. Wang Y, Mintz GS, Gu Z, Qi Y, Wang Y, Liu M and Wu X: Meta-analysis and systematic review of intravascular ultrasound versus angiography-guided drug eluting stent implantation in left main coronary disease in 4592 patients. BMC Cardiovasc Disord 18: 115, 2018.

40. Gori T, Polimeni A, Indolfi C, Räber L, Adriaenssens T and Münzel T: Predictors of stent thrombosis and their implications for clinical practice. Nat Rev Cardiol 16: 243-256, 2019.

41. Stone GW, Sabik JF, Serruys PW, Simonton CA, Généreux P, Puskas J, Kandzari DE, Morice MC, Lembo N, Brown WM III, et al: Everolimus-eluting stents or bypass surgery for left main coronary artery disease. N Engl J Med 375: 2223-2235, 2016. 
42. Hildick-Smith D, de Belder AJ, Cooter N, Curzen NP, Clayton TC, Oldroyd KG, Bennett L, Holmberg S, Cotton JM, Glennon PE, et al: Randomized trial of simple versus complex drug-eluting stenting for bifurcation lesions: The British bifurcation coronary study: Old, new, and evolving strategies. Circulation 121: 1235-1243, 2010.

43. Ferenc M, Gick M, Comberg T, Rothe J, Valina C, Toma A, Löffelhardt N, Hochholzer W, Riede F, Kienzle RP, et al: Culotte stenting vs TAP stenting for treatment of de-novo coronary bifurcation lesions with the need for side-branch stenting: The bifurcations bad krozingen (BBK) II angiographic trial. Eur Heart J 37: 3399-3405, 2016.

44. Prati F, Jenkins MW, Di Giorgio A and Rollins AM: Intracoronary optical coherence tomography, basic theory and image acquisition techniques. Int J Cardiovasc Imaging 27: 251-258, 2011.

45. Okamura T, Yamada J, Nao T, Suetomi T, Maeda T, Shiraishi K, Miura T and Matsuzaki M: Three-dimensional optical coherence tomography assessment of coronary wire re-crossing position during bifurcation stenting. EuroIntervention 7: 886-887, 2011.

46. Galassi AR, Sianos G, Werner GS, Escaned J, Tomasello SD, Boukhris M, Castaing M, Büttner JH, Bufe A, Kalnins A, et al: Retrograde recanalization of chronic total occlusions in Europe: Procedural, in-hospital, and long-term outcomes from the multicenter ERCTO registry. J Am Coll Cardiol 65: 2388-2400, 2015.

47. Munce NR, Yang VX, Standish BA, Qiang B, Butany J, Courtney BK, Graham JJ, Dick AJ, Strauss BH, Wright GA and Vitkin IA: Ex vivo imaging of chronic total occlusions using forward-looking optical coherence tomography. Lasers Surg Med 39: 28-35, 2007.

48. Tyczynski P, Ferrante G, Kukreja N, Moreno-Ambroj C, Barlis P, Ramasami N, De Silva R, Beatt K and Di Mario C: Optical coherence tomography assessment of a new dedicated bifurcation stent. EuroIntervention 5: 544-551, 2009.

49. Tanigawa J, Barlis P and Di Mario C: Heavily calcified coronary lesions preclude strut apposition despite high pressure balloon dilatation and rotational atherectomy: In-vivo demonstration with optical coherence tomography. Circ J 72: 157-160, 2008.

50. Cockburn J, Hildick-Smith D, Cotton J, Doshi S, Hanratty C, Ludman P, Robinson D, Redwood S, de Belder $M$ and de Belder A: Contemporary clinical outcomes of patients treated with or without rotational coronary atherectomy-an analysis of the UK central cardiac audit database. Int J Cardiol 170: 381-387, 2014.

51. Jaguszewski M, Aloysius R, Wang W, Bezerra HG, Hill J, De Winter RJ, Karjalainen PP, Verheye S, Wijns W, Lüscher TF, et al: The REMEDEE-OCT study: An evaluation of the bioengineered COMBO dual-therapy CD34 antibody-covered sirolimus-eluting coronary stent compared with a cobalt-chromium everolimus-eluting stent in patients with acute coronary syndromes: Insights from optical coherence tomography imaging analysis. JACC Cardiovasc Interv 10: 489-499, 2017.

52. Barlis P, Regar E, Serruys PW, Dimopoulos K, van der Giessen WJ, van Geuns RJ, Ferrante G, Wandel S, Windecker S, van Es GA, et al: An optical coherence tomography study of a biodegradable vs. durable polymer-coated limus-eluting stent: A LEADERS trial sub-study. Eur Heart J 31: 165-176, 2010.

53. Hamilos MI, Ostojic M, Beleslin B, Sagic D, Mangovski L, Stojkovic S, Nedeljkovic M, Orlic D, Milosavljevic B, Topic D, et al: Differential effects of drug-eluting stents on local endothelium-dependent coronary vasomotion. J Am Coll Cardiol 51: 2123-2129, 2008.

54. Brugaletta S, Heo JH, Garcia-Garcia HM, Farooq V, van Geuns RJ, de Bruyne B, Dudek D, Smits PC, Koolen J, McClean D, et al: Endothelial-dependent vasomotion in a coronary segment treated by ABSORB everolimus-eluting bioresorbable vascular scaffold system is related to plaque composition at the time of bioresorption of the polymer: Indirect finding of vascular reparative therapy? Eur Heart J 33: 1325-1333, 2012.

55. Kubo T, Imanishi T, Kitabata H, Kuroi A, Ueno S, Yamano T, Tanimoto T, Matsuo Y, Masho T, Takarada S, et al: Comparison of vascular response after sirolimus-eluting stent implantation between patients with unstable and stable angina pectoris: A serial optical coherence tomography study. JACC Cardiovasc Imaging 1: 475-484, 2008.

56. Kochman J, Pietrasik A, Rdzanek A, Ścibisz A, Pawlak M, Filipiak KJ and Opolski G: Tissue coverage of paclitaxel and sirolimus eluting stents in long term follow-up: Optical coherence tomography study. Cardiol J 20: 247-253, 2013.
57. Landmesser U, Engberding N, Bahlmann FH, Schaefer A, Wiencke A, Heineke A, Spiekermann S, Hilfiker-Kleiner D, Templin C, Kotlarz D, et al: Statin-induced improvement of endothelial progenitor cell mobilization, myocardial neovascularization, left ventricular function, and survival after experimental myocardial infarction requires endothelial nitric oxide synthase. Circulation 110: 1933-1939, 2004.

58. Kimura T, Abe K, Shizuta S, Odashiro K, Yoshida Y, Sakai K, Kaitani K, Inoue K, Nakagawa Y, Yokoi H, et al: Long-term clinical and angiographic follow-up after coronary stent placement in native coronary arteries. Circulation 105: 2986-2991, 2002.

59. Stone GW, Moses JW, Ellis SG, Schofer J, Dawkins KD, Morice MC, Colombo A, Schampaert E, Grube E, Kirtane AJ, et al: Safety and efficacy of sirolimus- and paclitaxel-eluting coronary stents. N Engl J Med 356: 998-1008, 2007.

60. Jung HW, Lim C, Bae HJ, Lee JH, Lee YJ, Kim JS, Lee SJ, Hong SJ, Ahn CM, Kim BK, et al: Association between in-stent neointimal characteristics and native coronary artery disease progression. PLoS One 16: e0247359, 2021.

61. Borovac JA, D'Amario D, Vergallo R, Porto I, Bisignani A, Galli M, Annibali G, Montone RA, Leone AM, Niccoli G and Crea F: Neoatherosclerosis after drug-eluting stent implantation: A novel clinical and therapeutic challenge. Eur Heart J Cardiovasc Pharmacother 5: 105-116, 2019.

62. Oka N, Kadohira T, Fujii K, Kitahara H, Fujimoto Y and Kobayashi Y: Microbubble contrast enhancement of neointima after drug-eluting stent implantation: An optical coherence tomography study. Heart Vessels 34: 393-400, 2019.

63. Li S, Luo C and Chen H: Risk factors of in-stent restenosis in patients with diabetes mellitus after percutaneous coronary intervention: A protocol for systematic review and meta-analysis. Medicine (Baltimore) 100: e25484, 2021.

64. Kang SJ, Song HG, Ahn JM, Kim WJ, Lee JY, Park DW, Lee SW, Kim YH, Lee CW, Park SW and Park SJ: OCT-verified neoatherosclerosis in BMS restenosis at 10 years. JACC Cardiovasc Imaging 5: 1267-1268, 2012.

65. Toutouzas K, Chatzizisis YS, Riga M, Giannopoulos A, Antoniadis AP, Tu S, Fujino Y, Mitsouras D, Doulaverakis C, Tsampoulatidis I, et al: Accurate and reproducible reconstruction of coronary arteries and endothelial shear stress calculation using 3D OCT: Comparative study to 3D IVUS and 3D QCA. Atherosclerosis 240: 510-519, 2015.

66. Kaivosoja TP, Liu S, Dijkstra J, Huhtala H, Sheth T and Kajander OA: Comparison of visual assessment and computer image analysis of intracoronary thrombus type by optical coherence tomography. PLoS One 13: e0209110, 2018.

67. Guagliumi G, Sirbu V, Musumeci G, Gerber R, Biondi-Zoccai G, Ikejima H, Ladich E, Lortkipanidze N, Matiashvili A, Valsecchi O, et al: Examination of the in vivo mechanisms of late drug-eluting stent thrombosis: findings from optical coherence tomography and intravascular ultrasound imaging. JACC Cardiovasc Interv 5: 12-20, 2012.

68. Gori T: Vascular wall reactions to coronary stents-clinical implications for stent failure. Life (Basel) 11: 63, 2021.

69. Garcia-Guimaraes $M$, Antuña $P$, De la Cuerda F, Maruri-Sanchez R, Cuesta J, Bastante T, Rivero F and Alfonso F: High-definition IVUS versus OCT to assess coronary artery disease and results of stent implantation. JACC Cardiovasc Imaging 13: 519-521, 2020.

70. Park KW, Park JJ, Chae IH, Seo JB, Yang HM, Lee HY, Kang HJ, Cho YS, Yeon TJ, Chung WY, et al: Clinical characteristics of coronary drug-eluting stent fracture: Insights from a two-center des registry. J Korean Med Sci 26: 53-58, 2011.

71. Aung SSM, Latt H, Kyaw K and Roongsritong C: An interesting case and literature review of a coronary stent fracture in a current generation platinum chromium everolimus-eluting stent. Case Rep Cardiol 2018: 4579184, 2018.

72. Kan J, Ge Z, Zhang JJ, Liu ZZ, Tian NL, Ye F, Li SJ, Qian XS, Yang S, Chen MX, et al: Incidence and clinical outcomes of stent fractures on the basis of 6,555 patients and 16,482 drug-eluting stents from 4 centers. JACC Cardiovasc Interv 9: 1115-1123, 2016.

73. Kuramitsu S, Iwabuchi M, Yokoi H, Domei T, Sonoda S, Hiromasa T, Morinaga T, Kobayashi Y, Ohe K, Goya K, et al: Incidence and clinical impact of stent fracture after the Nobori biolimus-eluting stent implantation. J Am Heart Assoc 3: e000703, 2014. 
74. Shaw LJ, Berman DS, Maron DJ, Mancini GB, Hayes SW, Hartigan PM, Weintraub WS, O'Rourke RA, Dada M, Spertus JA, et al: Optimal medical therapy with or without percutaneous coronary intervention to reduce ischemic burden: Results from the clinical outcomes utilizing revascularization and aggressive drug evaluation (COURAGE) trial nuclear substudy. Circulation 117: 1283-1291, 2018.

75. Yin J, Li X, Jing J, Li J, Mukai D, Mahon S, Edris A, Hoang K, Shung KK, Brenner M, et al: Novel combined miniature optical coherence tomography ultrasound probe for in vivo intravascular imaging. J Biomed Opt 16: 060505, 2011.

76. Li BH, Leung AS, Soong A, Munding CE, Lee H, Thind AS, Munce NR, Wright GA, Rowsell CH, Yang VX, et al: Hybrid intravascular ultrasound and optical coherence tomography catheter for imaging of coronary atherosclerosis. Catheter Cardiovasc Interv 81: 494-507, 2013.

77. Li X, Li J, Jing J, Ma T, Liang S, Zhang J, Mohar D, Raney A, Mahon S, Brenner M, et al: Integrated IVUS-OCT imaging for atherosclerotic plaque characterization. IEEE J Sel Top Quantum Electron 20: 7100108, 2014.
78. Yoo H, Kim JW, Shishkov M, Namati E, Morse T, Shubochkin R, McCarthy JR, Ntziachristos V, Bouma BE, Jaffer FA and Tearney GJ: Intra-arterial catheter for simultaneous microstructural and molecular imaging in vivo. Nat Med 17:1680-1684, 2011.

79. Ligthart JM, Diletti R, Witberg $K$ and Schultz C: Three-dimensional optical coherence tomography for guidance of complex percutaneous coronary interventions. JACC Cardiovasc Interv 7: 102-103, 2014.

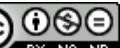

This work is licensed under a Creative Commons Attribution-NonCommercial-NoDerivatives 4.0 International (CC BY-NC-ND 4.0) License. 